\title{
Synthesis of the Polyhydroquinoline Derivative 4-(2- Chloro-Phenyl)-2,7,7-Trimethyl5-oxo-1,4,5,6,7,8- Hexahydroquinoline-3-Carboxylic Acid Ethyl Ester: Antimicrobial and Enzyme Modulator
}

Gustavo Henrique Andrade Machado ( $\nabla$ andradegh@gmail.com )

Universidade Federal de Lavras https://orcid.org/0000-0003-2900-9714

Marcus Vinícius Cardoso Trento

UFLA: Universidade Federal de Lavras

Juliana Junqueira Pinelli

UFLA: Universidade Federal de Lavras

Roberta Hilsdorf Piccoli

UFLA: Universidade Federal de Lavras

Sérgio Scherrer Thomasi

UFLA: Universidade Federal de Lavras

Silvana Marcussi

UFLA: Universidade Federal de Lavras

\section{Research Article}

Keywords: Multicomponent reactions, Chemical Characterization, Green Chemistry, Biological activities, anti-inflammatory, anticoagulant.

Posted Date: September 14th, 2021

DOI: https://doi.org/10.21203/rs.3.rs-884356/v1

License: (c) (i) This work is licensed under a Creative Commons Attribution 4.0 International License.

Read Full License 
Synthesis of the polyhydroquinoline derivative 4-(2-chloro-phenyl)-2,7,7-trimethyl-

5-oxo-1,4,5,6,7,8-hexahydroquinoline-3-carboxylic acid ethyl ester: antimicrobial and enzyme modulator

Gustavo Henrique Andrade Machado ${ }^{1}$, Marcus Vinicius Cardoso Trento ${ }^{1}$, Juliana Junqueira Pinelli ${ }^{2}$, Roberta Hilsdorf Piccoli ${ }^{2}$, Sergio Scherrer Thomasi ${ }^{1}$, Silvana Marcussi $^{1}$

${ }^{1}$ Department of Chemistry. Universidade Federal de Lavras (UFLA), Campus Universitário, Lavras, MG, Brazil, PO Box 3037, Zip code 37200-000, Phone no. $+55(35) 3829-1893$.

${ }^{2}$ Department of Food Sciences. Universidade Federal de Lavras (UFLA), Campus Universitário, Lavras, MG, Brazil, Zip code 37200-000.

Corresponding author: MSc. Gustavo Henrique Andrade Machado. Department of Chemistry, Biochemistry Laboratory. Universidade Federal de Lavras (UFLA), Campus Universitário, Lavras, MG, Brazil, PO Box 3037, Zip Code 37200-000, phone no. $+55(35) 3829-1893$. 


\begin{abstract}
Multicomponent reactions are extremely relevant in green chemistry. They offer better conditions than traditional synthesis and are, therefore, used for many organic modifications. Recently, the synthesis of polyhydroquinolines has received much attention for its high pharmacological potential. In the present study, a polyhydroquinoline derivative was synthesized without the use of catalysts or solvents. The results of nuclear magnetic resonance and infrared spectroscopy demonstrated that the molecule was successfully synthesized. The molecule presents significant results of antimicrobial activity for the bacteria tested in the serial dilution method. It also increased the clotting time by 25.66 seconds for the highest dose and 12.66 seconds for the other doses tested. Prior incubation with the dose of $125 \mathrm{mg}$ reduced the thrombolytic activity to $73 \%$. The 125,100 , and $50 \mathrm{mg}$ doses previously incubated with Bothrops moojeni venom inhibited approximately $30 \%$ of the phospholipase activity. The molecule was also able to reduce the cytotoxicity induced by proteases significantly. In conclusion, the molecule presents several biological properties, which highlights its pharmaceutical potential.
\end{abstract}

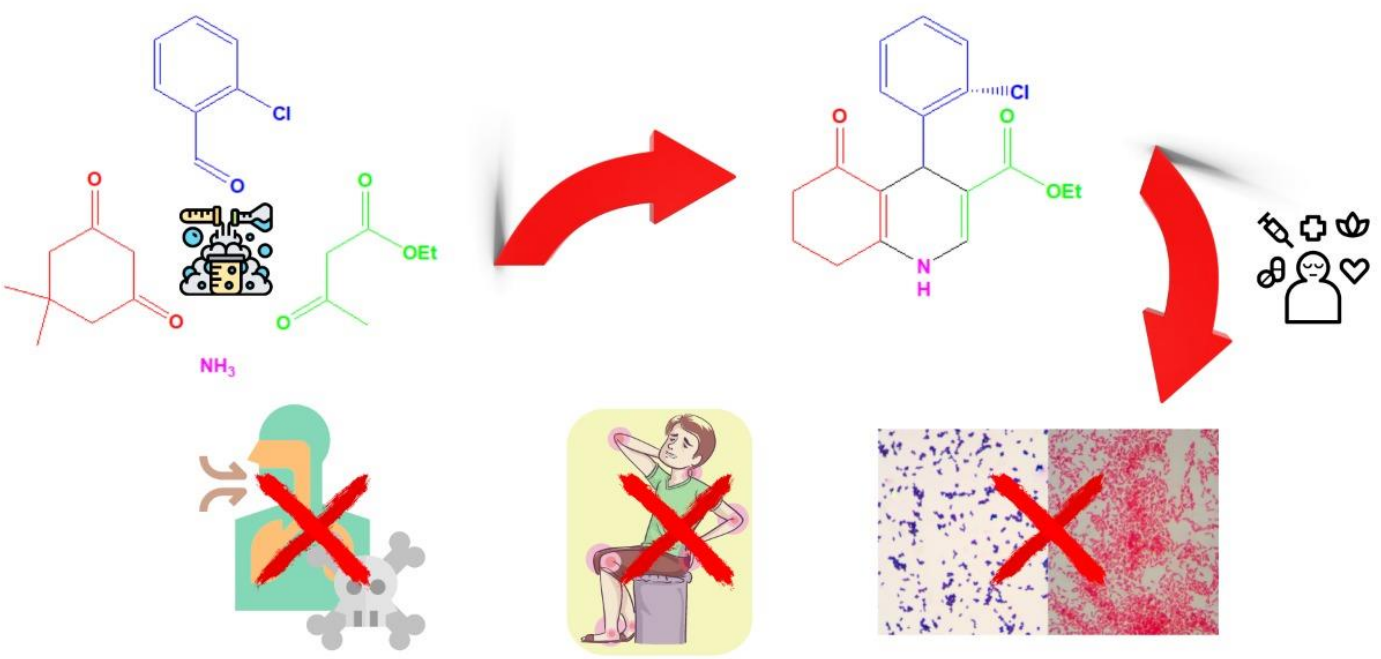


Keywords: Multicomponent reactions, Chemical Characterization, Green Chemistry, Biological activities, anti-inflammatory, anticoagulant.

\section{INTRODUCTION}

Green chemistry is based on environmental factors and arose from a natural need to adapt previously performed techniques. In order to develop environmentally-friendly strategies that minimize waste production and avoid the use of hazardous chemicals, green chemistry has been used in the pharmaceutical, food, and chemical industries $(1,2)$.

Multicomponent reaction (MCR) corresponds to convergent reactions in which three or more starting materials react in a single step to form a product. In discovering and developing new drugs, MCRs offer several advantages over traditional synthesis, such as shorter experimentation time, fewer laboratory techniques and solvents used, and the generation of smaller amounts of waste (3). Thus, the multicomponent reaction method is in accordance with the principles of green chemistry.

Numerous methods have been reported for the synthesis of polyhydroquinoline derivatives (PHQs). The classical method involves coupling three components under reflux: aldehyde, ethyl acetoacetate, and ammonium acetate in acetic acid or alcohol. However, new techniques to obtain these derivatives have been developed and studied. Said methods seek greater efficiency and yield, using reagents that can be recycled and are less toxic $(4,5)$.

PHQs has emerged as one of the most important compounds for the treatment of cardiovascular diseases (e.g., hypertension). These compounds have different medicinal functions, including neuroprotection, platelet aggregation inhibitor, antimicrobial, antiischemic agent, and potentiators in chemotherapy $(5,6)$.

Therefore, the objective in the present work was to carry out the multicomponent reaction of a PHQ derivative and perform its structural characterization using the Fourier transform infrared spectroscopy (FTIR) and ${ }^{1} \mathrm{H}$ - and ${ }^{13} \mathrm{C}$ - nuclear magnetic resonance (NMR). In addition, the biological activities exerted by the synthesized compound were also evaluated (antimicrobial activity and modulatory potential on enzymes that act in hemostatic and inflammatory processes). 


\section{MATERIAL AND METHODS}

\subsection{Characterization by Nuclear Magnetic Resonance (NMR)}

The NMR spectra were obtained at the Universidade Federal de São Carlos (UFSCar) using a Bruker Avance III - 14.1 Tesla (600.23 MHz for $1 \mathrm{H}$ frequency) with an Ultrashield Plus ${ }^{\circledR}$ magnet. The spectrometer is equipped with a $5 \mathrm{~mm}$ TCI (Triple

Resonance Inverse) CryoProbe $-{ }^{1} \mathrm{H}$ and ${ }^{13} \mathrm{C}$ nuclei- and cold preamplifiers (temperature at approximately $77 \mathrm{~K})$. The probe has a Z-Gradient $(53 \mathrm{G} / \mathrm{cm})$ and an Automatic Tuning and Matching accessory (ATMA).

\subsection{Attenuated Total Reflection- Fourier transform infrared (FTIR/ATR) spectroscopy}

The ATR infrared spectra were obtained using a Varian equipment coupled to a diamond ATR accessory (GladiATR - PIKE Technologies), with a spectral range of 4000 to $400 \mathrm{~cm}^{-1}$ and resolution of 2000 to $4 \mathrm{~cm}^{-1}$.

The analysis of the functional groups in the synthesized molecule was performed by Fourier transform infrared spectroscopy (FTIR). The IR absorption spectra were obtained with $\mathrm{KBr}$ pellets, where $2.0 \mathrm{mg}$ of the sample was added into $200.0 \mathrm{mg}$ of $\mathrm{KBr}$. The analyses were performed at the Chemical Analysis and Prospection Center (CAPQ), located at the Department of Chemistry/UFLA.

\subsection{Evaluation of the Antimicrobial Activity}

The antimicrobial activity of the molecule 4-(2-chloro-phenyl)-2,7,7-trimethyl-5oxo-1,4,5,6,7,8-hexahydroquinoline-3-carboxylic acid ethyl ester was evaluated by susceptibility testing using the agar diffusion method (antibiogram) and pour-plate technique with serial dilution (broth microdilution), according to the recommendations of the CLINICAL AND LABORATORY STANDARDS INSTITUTE (7).

\subsubsection{Obtaining the microorganisms and inoculum}

The microorganisms used were Staphylococcus aureus ATCC 25923 and Pseudomonas aeruginosa ATCC 27853, which were obtained from Fundação Oswaldo Cruz (FIOCRUZ), Rio de Janeiro, Brazil. These strains were chosen because of their presence in wounds and interference in the healing process. The stock cultures were stored in a cell freezing medium (glycerol: $15 \mathrm{~mL}$; peptone, bacteriological: $0.5 \mathrm{~g}$; yeast extract: $0.3 \mathrm{~g}$; $\mathrm{NaCl}: 0.5 \mathrm{~g}$; distilled water: $100 \mathrm{~mL}$ ) and kept frozen during the 
experiment. The reactivation of the strains was performed under aerobic conditions by inoculating $100 \mu \mathrm{L}$ of the culture into tubes containing $10 \mathrm{~mL}$ of Brain Heart Infusion (BHI) broth for $24 \mathrm{~h}$ at $37^{\circ} \mathrm{C}$. The inoculum was standardized (McFarland standards) to a cell density corresponding to $10^{8} \mathrm{CFU} / \mathrm{mL}$.

\subsubsection{Antimicrobial testing}

For the antibiogram, aliquots of the $S$. aureus and $P$. aeruginosa cultures were spread on Petri dishes containing Trypticase Soy Agar (TSA). Disks with the molecule 4-(2-chloro-phenyl)-2,7,7-trimethyl-5-oxo-1,4,5,6,7,8-hexahydroquinoline-3-carboxylic acid ethyl ester in the doses of $500 \mathrm{mg}, 250 \mathrm{mg}, 125 \mathrm{mg}$, and $100 \mathrm{mg}$ were added to the medium. The dishes were incubated at $37{ }^{\circ} \mathrm{C}$ for $24 \mathrm{~h}$. After that period, inhibition halos were measured around the disks. The antibiotic Chloramphenicol (8) was used as a positive control $(30 \mu \mathrm{g})$.

A solution containing $500 \mathrm{mg}$ of the compound diluted in $1 \mathrm{~mL}$ DMSO was used for broth microdilution. $500 \mu \mathrm{L}$ of this solution were incubated with $500 \mu \mathrm{L}$ of BHI broth for 48 hours (stock solution), obtaining a final dose of $250 \mathrm{mg}$. The cell count was performed three times: 0 hours (preparation of the incubation), 24 hours, and 48 hours. Dilutions ( $900 \mu \mathrm{L}$ of distilled water for each $100 \mu \mathrm{L}$ of the stock solution) were performed in Eppendorf tubes to count the viable cells. Petri dishes containing TSA were incubated at $37^{\circ} \mathrm{C}$ for $24 \mathrm{~h}$ with $S$. aureus and $37^{\circ} \mathrm{C}$ for $48 \mathrm{~h}$ with $P$. aeruginosa. The spread plate technique was performed at 0,24 , and 48 hours, with serial dilutions to obtain an adequate number of colonies (7). S. aureus or P. aeruginosa in $1000 \mu \mathrm{L}$ of BHI were used as positive controls, which were performed at 0,24 , and 48 hours.

Both tests and all concentrations were evaluated in triplicate.

\subsection{Obtaining human biological material}

Experimental protocols (coagulation, cytotoxicity on erythrocytes, and thrombolytic) that require the collection and use of human blood were previously approved by the Committee for Ethics in Research on Human Beings (COEP) of the Universidade Federal de Lavras (registration number: 10991019.4.0000.5148/ 3.288.976).

The volunteers (between 18 and 35 years old) were non-smokers and declared that they did not use prescription medication, nor did they work under chemical or biological risk conditions. 


\subsection{Effects on the coagulation of citrated human plasma}

The coagulation activity was evaluated following the methodology described by MOURAO et al. (9). Human plasma was collected on a citrate tube (200 $\mu \mathrm{L})$ and stabilized at $37^{\circ} \mathrm{C}$. Treatments were added to the tube, and the time until the formation of a rigid clot was measured. The potential to inhibit coagulation was evaluated by adding different doses of the compound to the citrated plasma and incubating them for 10 minutes. After that, $40 \mu \mathrm{g}$ of Bothrops moojeni venom was added to the tube, and the clotting time was measured. This experiment allows the observation of possible interactions between the compound and proteins of the coagulation cascade.

In addition, the assay was also performed by incubating the compound at different doses with the venom and only then adding citrated plasma. The results obtained allow the observation of possible interactions between the compound and serine proteases present in the venom.

All treatments were evaluated in triplicates.

\subsection{Effects on human blood thrombi and venom-induced thrombolytic activity}

The thrombolytic activity was evaluated on blood clots formed in vitro, according to the methodology described by CINTRA et al. (10). Thrombi were obtained by applying $100 \mu \mathrm{L}$ of freshly collected blood (without anticoagulant) into the wells of a 96-well microplate. The treatments were added to the thrombi in triplicates, and then the microplates remained in a cell culture chamber for 24 hours at $36^{\circ} \mathrm{C}$. The results were evaluated by measuring the volume of liquids released by the thrombi. The compound was evaluated at different doses. The same doses were also previously incubated with Bothrops moojeni $(20 \mu \mathrm{g})$ venom for 30 minutes at $37^{\circ} \mathrm{C}$ to assess their activity on thrombolytic proteases. $30 \mu \mathrm{L}$ of phosphate-buffered saline (PBS) was used as the negative control, which corresponds to the same volume as the treatments. Bothrops moojeni $(20 \mu \mathrm{g})$ was used as the positive control. The results were converted into percentages, and the positive control was considered as $100 \%$ of activity.

\subsection{Effects on the activity of phospholipases $A_{2}$}

The phospholipase activity was evaluated according to the methodology described by GUTIÉRREZ et al. (11), with few adaptions. A medium was formed with $0.01 \mathrm{~mol} \mathrm{~L}^{-}$ $1 \mathrm{CaCl}_{2}$, egg yolk phospholipids (phosphatidylcholine, phosphatidylserine, and phosphatidylethanolamine), $0.005 \%$ sodium azide (to prevent the growth of 
microorganisms in the medium), and 1\% bacteriological agar dissolved in PBS (pH 7.2). The medium was poured into Petri dishes and, after solidification, the samples were applied to holes made in the gel $(\sim 0.5 \mathrm{~cm}$ diameter). The dishes were kept in a cell culture chamber at $36^{\circ} \mathrm{C}$ for 18 hours, and the activity was evaluated by measuring the translucent halo formed around the hole (in millimeters - $\mathrm{mm}$ ). The anti-inflammatory potential of the compound was evaluated by observing its effects on the activity of phospholipases $\mathrm{A}_{2}$ present in Bothrops moojeni venom. Thus, the compound was previously incubated in different doses with the venom $(20 \mu \mathrm{g})$, for 30 minutes at $37^{\circ} \mathrm{C}$, with subsequent evaluation of the phospholipase activity. Positive controls were performed using only the venom. All treatments were evaluated in triplicates.

\subsection{Cytotoxic activity on human erythrocytes}

This activity was evaluated using a solid medium, as previously described by Gutiérrez et al. (11). However, the phospholipids were replaced by human erythrocytes. The blood collected in tubes containing heparin was immediately centrifuged, and the supernatant discarded. $10 \mathrm{~mL}$ of PBS was added to the erythrocytes, which were washed twice more in PBS with centrifugations at $1200 \mathrm{~g}$ for 10 minutes. The obtained erythrocytes concentrate $\left(1: 3 \mathrm{v} \mathrm{v}^{-1}\right.$ in PBS) was used to compose the gel, which also included $0.01 \mathrm{~mol} \mathrm{~L}^{-1} \mathrm{CaCl}_{2}, 0.005 \%$ sodium azide, and $1 \%$ bacteriological agar dissolved in PBS ( $\mathrm{pH} 7.2$ ). After solidification, the treatments were applied to $\sim 0.5 \mathrm{~cm}$ diameter holes made in the gel. The Petri dishes were kept in a cell culture chamber at $36^{\circ} \mathrm{C}$ for 18 hours. The formation of a translucent halo around the hole characterizes hemolysis, which was measured (millimeters) with a caliper. The compound was evaluated in different doses to observe if it induced erythrocyte lysis. Then, to verify its anti-cytotoxic potential, the compound was evaluated after pre-incubation with B. moojeni venom $(20 \mu \mathrm{g})$ for 30 minutes at $36^{\circ} \mathrm{C}$. B. moojeni venom was used as a positive control. All treatments were evaluated in triplicates.

\subsection{Statistical Analysis}

The data obtained were submitted to the Shapiro-Wilk test at a 5\% significance level to verify their normality. Dunnett's test was performed for the data that had a normal distribution. A normalization attempt was made using the RANK procedure (PROC RANK) on the data that did not show a normal distribution. Non-normalized data were 
compared by the Kruskal-Wallis test at a 5\% significance level. The statistical software used was SAS (version 9.0).

\section{RESULTS AND DISCUSSION}

\subsection{Synthesis and Structural Characterization}

The hexahydroquinoline derivative was synthesized according to the methodology described by Kumar et al. (12), which did not use catalysts and solvents. The following reagents were used in the reaction (Figure 1): 5,5- dimethyl -1,3cyclohexanedione $(2 \mathrm{mmol}), 2$-chlorobenzaldehyde $(2 \mathrm{mmol})$, ethyl acetoacetate (2 $\mathrm{mmol})$, and ammonium acetate $(3 \mathrm{mmol})$.

The mechanism of the general reaction for obtaining PHQs derivatives (13) begins with the removal of an $\alpha$-hydrogen from dimedone by a base, forming an enolate ion that has an area of a high concentration negative charge on the $\alpha$-carbon. This compound is stabilized by the resonance between the electron pairs and the carbonyl groups bonded to the carbon. These electrons on the $\alpha$-carbon are the ones that attack the carbon of the carbonyl group of benzaldehyde, which has a positive charge density because of the inductive effect caused by the oxygen. The oxygen on the carbonyl group acquires a negative charge after the nucleophilic attack since it breaks one of its bonds with the carbon that was attacked. The carbon captures a hydrogen from the reaction components and forms a secondary alcohol to stabilize this excess negative charge.

The compound still has another $\alpha$-hydrogen that can be removed by the base present in the reaction. The electron pair that formed the $\mathrm{C}-\mathrm{C}$ bond now forms a $\mathrm{C}=\mathrm{C}$ double bond, eliminating the hydroxyl group. Although the -OH group is an unfavorable leaving group, its elimination in this synthesis step is justified by the high stability of the product formed - an $\alpha, \beta$-unsaturated carbonyl compound. The electrons of the new double bond can perform resonance with both the carbonyl groups and the aromatic ring $(14,15)$ observed in Figure 1.

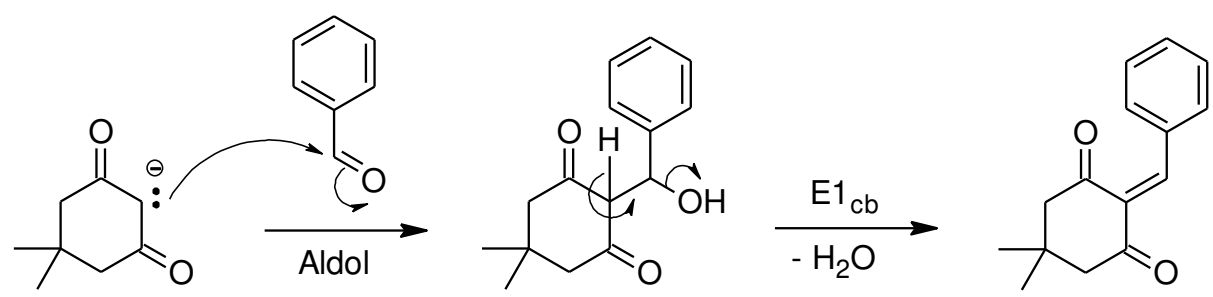

Figure 1. Aldol condensation between dimedone and benzaldehyde. 
Since the electrons of the double bond can perform resonance with the carbonyl groups, the $\beta$-carbon gets a positive charge density and becomes an electrophilic center. Another enolate ion, formed from ethyl acetoacetate, reacts with the compound previously formed by a Michael addition. After the attack, the oxygen in the dimedone acquires a negative charge that is stabilized by capturing hydrogen and forming a $-\mathrm{OH}$. However, the $\mathrm{C}=\mathrm{O}$ double bond is repaired by tautomerism, and the negative charge passes to the neighboring carbon, which is stabilized by a hydrogen bond. This compound then reacts with ammonia to form an imine.

The mechanism begins with the protonation of the carbonyl group. Consequently, the inductive effect caused by the now positively charged oxygen increases, enabling the attack of the ammonia electron pairs on the carbon of the protonated carbonyl group. After the charges are stabilized, amino alcohol is formed. Then, the nitrogen can make a $\mathrm{C}=\mathrm{N}$ double bond and force out the $-\mathrm{OH}$ group in the form of water, carrying one of its hydrogens and finally forming imine (15), as per Figure 2.<smiles>CCOC(=O)C(=O)C(C)=O</smiles>

Figure 2. Michael addition between ethyl acetoacetate and the product of the aldol condensation, followed by imine formation.

The imine can still tautomerize and get into equilibrium with an enamine. Due to the inductive effect, the carbon bonded to the ester's carbonyl group and imine has an excessive acidity. Therefore, it can be removed by a base from the reaction, which forms the double bond of the enamine. The electrons of the $\mathrm{C}=\mathrm{N}$ double bond are concentrated on the nitrogen, which stabilizes this charge by bonding to hydrogen (15), as shown in Figure 3. 
<smiles>CCOC(=O)C(C(C)=N)C(c1ccccc1)C1C(=O)CC(C)(C)CC1=O</smiles>

Imina<smiles>C#CC1(C)CC(=O)C(C(C(C(=O)OCC)=C(C)N)c2ccccc2)C(=O)C1</smiles>

Enamina

Figure 3. Chemical equilibrium between imine and enamine.

Due to their proximity, the free electrons of the nitrogen can attack the carbon of the carbonyl group of dimedone. This attack breaks the $\mathrm{C}=\mathrm{O}$ double bond and places the electrons on the oxygen, which is stabilized by a proton transfer with one of the hydrogens of the amine, forming a hydroxyl group. Afterwards, nitrogen's lone pair can form a double bond by removing the hydroxyl group, which leaves a positive charge. This charge is stabilized by forming a double bond in the central ring with the removal of hydrogen. Once more, removing the hydroxyl group is favorable because of the possibility of resonance in the double bond formed (15). This final step for the synthesis of hexahydroquinoline is demonstrated in Figure 4.<smiles>CCOC(=O)C(C(=O)OC)=C(C)NCC1C(=O)CC(C)(C)CC1(C)O</smiles><smiles>CCOC(=O)C1=C(C)NC2(O)CC(C)(C)CC(=O)C1C2c1ccccc1</smiles><smiles>CCOC(=O)C1=C(C)NC2=C(C(=O)CC(C)(C)C2)C1c1ccccc1</smiles>

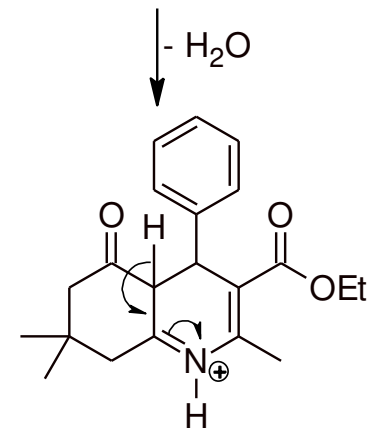

Figure 4. The final step in the mechanism for the hexahydroquinoline synthesis.

${ }^{1} \mathrm{H}-$ and ${ }^{13} \mathrm{C}-\mathrm{NMR}$ and IR were performed to characterize the derivative, which used 2-chlorobenzaldehyde as the aromatic aldehyde. In this case, a chlorine atom is expected to be connected in an ortho position in relation to the nitrogen ring. The ${ }^{1} \mathrm{H}-$ NMR spectrum shows resonances in $\delta_{\mathrm{H}} 5.38(\mathrm{H} 4,1 \mathrm{H}, s), 4.04\left(\mathrm{H} 12,2 \mathrm{H}, q,{ }^{3} J_{\mathrm{H} 12-\mathrm{H} 13}=7.0\right.$ $\mathrm{Hz}), 2.33-2.09(\mathrm{H} 7$ and $\mathrm{H} 9,4 \mathrm{H}, m), 2.30(\mathrm{H} 1,3 \mathrm{H}, s), 1.17\left(\mathrm{H} 13,3 \mathrm{H}, t,{ }^{3} J_{\mathrm{H} 13-\mathrm{H} 12}=7.0\right.$ 
$\mathrm{Hz}), 1.06(\mathrm{H} 8$ ', 3H, s) and $0.94(\mathrm{H} 8$ ', $3 \mathrm{H}, s)$, which is expected for this class of molecule. In the olefinic and aromatic region, four well-separated and defined multiplets that integrate to 4 hydrogen atoms confirm the presence of a disubstituted aromatic ring: $\delta_{\mathrm{H}}$ 7.40-7.38 (H19, 1H, $m)$, 7.27-7.22 (H16, 1H, m), 7.13-7.11 (H17, 1H, $m)$, and 7.04-7.01 $(\mathrm{H} 18,1 \mathrm{H}, m) \mathrm{ppm}$.

It is possible to identify the $\delta_{\mathrm{C}} 133.2(\mathrm{C} 15) \mathrm{ppm}$ signal in the ${ }^{13} \mathrm{C}$ NMR spectrum, which is compatible with the $s p^{2}$ hybridized carbon of the aromatic ring connected directly to a chlorine atom. The $\delta_{\mathrm{C}} 143.6(\mathrm{C} 14) \mathrm{ppm}$ signal is also observed, according to the non-hydrogenated $s p^{2}$ hybridized carbon of the aromatic ring neighboring the chlorinated carbon. The $\mathrm{C} 16, \mathrm{C} 17, \mathrm{C} 18$, and $\mathrm{C} 19$ carbons have their resonance in $\delta_{\mathrm{C}}$ 132.1, 129.6, 126.2, and 127.2, respectively. The other peaks observed also corroborate to the structural characterization: $\delta_{\mathrm{C}} 195.3$ (C6), 167.4 (C11), 148.89 (C2), 111.1 (C5), 105.3 (C3), 59.8 (C12), 50.6 (C7), 41.1 (C9), 36.0 (C4), 32.5 (C8), 29.3 (C8'), 27.2 (C8'), $19.2(\mathrm{C} 1)$, and 14.2 (C13) ppm.

Stretches were observed in the infrared (IR) spectrum of the synthesized compound: primary amine N-H stretch in $3296 \mathrm{~cm}^{-1}, \mathrm{C}_{s p 2}-\mathrm{H}$ stretch in $3068 \mathrm{~cm}^{-1}, \mathrm{C}_{s p 3}-\mathrm{H}$ stretch in 2954 and $2931 \mathrm{~cm}^{-1}$, ester $\mathrm{C}=\mathrm{O}$ stretch in $1613 \mathrm{~cm}^{-1}$ (more weakly bonded carbonyl group due to resonance), ketone $\mathrm{C}=\mathrm{O}$ stretch in $1696 \mathrm{~cm}^{-1}$, aromatic $\mathrm{C}=\mathrm{C}$ stretch in $1485 \mathrm{~cm}^{-1}$, and asymmetrical stretch of C-O-C (ester) in 1207 and $1068 \mathrm{~cm}^{-1}$. The band in $772 \mathrm{~cm}^{-1}$ probably refers to the stretching of the $\mathrm{C}-\mathrm{Cl}$ bond.

\subsection{Biological Activities}

\section{Antimicrobial activity}

No halos were formed in the antibiogram (agar diffusion test), which may be a result of the compound's inability to diffuse into the solid culture medium. However, when evaluated by the pour-plate technique with serial dilution (broth microdilution) in a liquid medium, the compound showed significant antimicrobial activity for both $S$. aureus and $P$. aeruginosa, as seen in Tables 1 and 2 . 


\begin{tabular}{cccccc}
\hline \multicolumn{5}{c}{ Antimicrobial activity } \\
\cline { 2 - 6 } & Time (h) & (Colony Forming Units, CFU) \\
\hline & Control & Compound & CV (\%) & $\begin{array}{c}\text { P- } \\
\text { value }\end{array}$ \\
\hline$*$ & 0 & 5.12 & 5.27 & 3.91 & 0.624 \\
$*$ & 24 & 11.99 & 4.04 & 54.59 & 0.033 \\
& 48 & 16.92 & 0.00 & 109.62 & 0.011 \\
\hline
\end{tabular}

Table 1. Effect of the compound 4-(2-chloro-phenyl)-2,7,7-trimethyl-5-oxo-1,4,5,6,7,8hexahydroquinoline-3-carboxylic acid ethyl ester and positive control on the viability and replication of Staphylococcus aureus ATCC 25923. CV (\%): Coefficient of Variation. Pvalue less than 0.05 is statistically significant. * Data have a normal distribution.

\begin{tabular}{cccccc}
\hline \multicolumn{5}{c}{ Antimicrobial activity } \\
\cline { 2 - 6 } & Time (h) & \multicolumn{4}{c}{ (Colony Forming Units, CFU) } \\
\hline & Control & Compound & CV (\%) & $\begin{array}{c}\text { P- } \\
\text { value }\end{array}$ \\
\hline$*$ & 0 & 4.67 & 4.75 & 3.31 & 0.574 \\
$*$ & 24 & 12.31 & 0.00 & 109.55 & 0.011 \\
& 48 & 18.41 & 0.00 & 109.70 & 0.011 \\
\hline
\end{tabular}

Table 2. Effect of the compound 4-(2-chloro-phenyl)-2,7,7-trimethyl-5-oxo-1,4,5,6,7,8hexahydroquinoline-3-carboxylic acid ethyl ester and positive control on the viability and replication of Pseudomonas aeruginosa ATCC 27853. CV (\%): Coefficient of Variation. P-value less than 0.05 is statistically significant. * Data have a normal distribution.

As observed in the tables above, there is no significant difference at time zero for both bacteria. Since the Petri dishes containing TSA are incubated exactly when the dilutions are performed (time zero), there is not enough time for the bacteria to interact with the synthesized molecule. However, when they are incubated after 24 and 48 hours with the treatment, significant inhibitory results are observed for both $S$. aureus and $P$. aeruginosa.

$S$. aureus has a decrease in the CFU number after $24 \mathrm{~h}$ in the presence of the compound. After $48 \mathrm{~h}$, there are no more colonies grown. A total absence of colony growth was observed after $24 \mathrm{~h}$ of treatment in P. aeruginosa, and the same results are observed after 48h. Therefore, the molecule 4-(2-chloro-phenyl)-2,7,7-trimethyl-5-oxo- 
1,4,5,6,7,8-hexahydroquinoline-3-carboxylic acid ethyl ester shows antimicrobial activity for both the Gram-positive and Gram-negative bacteria evaluated. These are promising data, considering the relevance and necessity of developing new drugs with broad-spectrum antimicrobial activity.

The synthesized molecule proves to be more efficient against Gram-negative bacteria, which are more challenging to treat (16). Thus, it is essential to study the class of polyhydroquinolines and their various biological activities.

Jamale et al. (17) showed that their polyhydroquinoline derivatives had significant potential as an antimicrobial. Some of the compounds were effective against Mycobacterium tuberculosis, which is an extremely difficult microorganism to eradicate. The derivatives were tested at $0.8,1.6,3.2,6.25,12.5,25,50$, and $100 \mu \mathrm{g} / \mathrm{mL}$ on the strain M. tuberculosis H37Rv ATCC 27294 to determine the minimum inhibitory concentration (MIC). Two synthesized compounds exhibited the most promising anti-TB activity, with a MIC of $1.6 \mu \mathrm{g} / \mathrm{mL}$. They were considered more potent than the standard drugs used as positive controls (pyrazinamide, streptomycin, and ciprofloxacin).

Recently (18), various polyhydroquinoline derivatives were evaluated to determine the minimum inhibitory concentration on Gram-positive (Bacillus subtilis) and Gram-negative (Escherichia coli) bacteria, filamentous fungi (Aspergillus niger), and yeast (Saccharomyces cerevisiae). According to the authors, some derivatives had significant antimicrobial activity, especially those with nitro, chlorine, bromine, and fluorine as the substituted functional group in the aromatic aldehyde. Their results corroborate the findings in the present study. Therefore, polyhydroquinoline derivatives can be promising molecules in the development of antimicrobial drugs.

\section{Effects on the coagulation of citrated human plasma}

The synthesized compound did not induce plasma coagulation in the tested doses (data not shown). When incubated with Bothrops moojeni venom $(40 \mu \mathrm{g})$, the compound prolonged the clotting time. However, when compared to the positive control, a statistically significant difference was observed only for the higher dose - $125 \mathrm{mg}$ (Figure $5 \mathrm{~A})$. In contrast, when the venom is added later to plasma previously incubated with the compound, a protective effect was observed at 125,100 , and $50 \mathrm{mg}(\mathrm{p}<0.05)$. In this case, there was a significant increase in the clotting time (25.66 seconds for the $125 \mathrm{mg}$ dose and 12.66 seconds for the 100 and $50 \mathrm{mg}$ doses) (Figure 5B). 

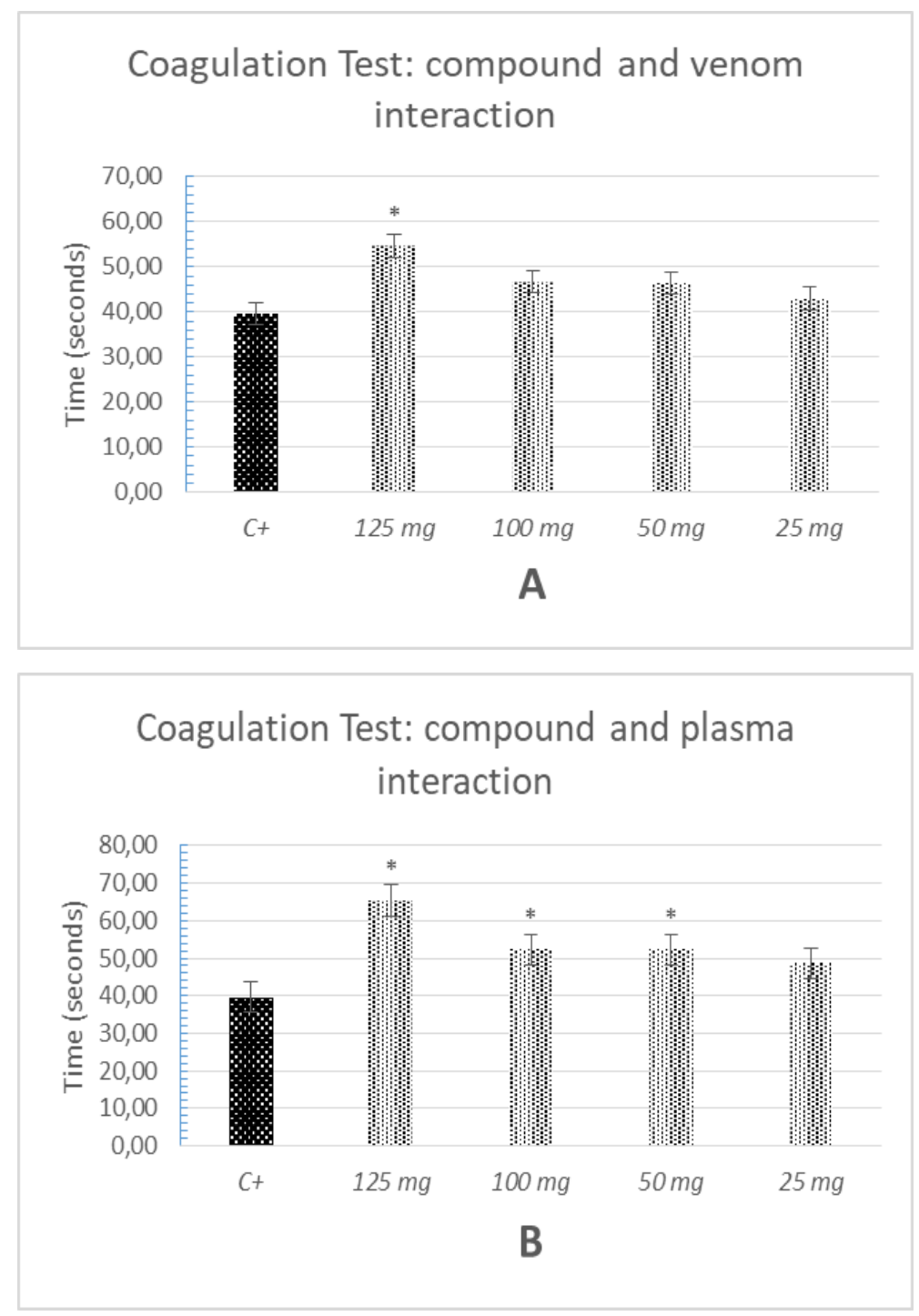

Figure 5. (A) Effect of the compound 4-(2-chloro-phenyl)-2,7,7-trimethyl-5-oxo1,4,5,6,7,8-hexahydroquinoline-3-carboxylic acid ethyl ester on the coagulation (induced by the venom of Bothrops moojeni) of citrated human plasma. Positive control $(\mathrm{C}+)$ : Bothrops moojeni venom $(40 \mu \mathrm{g})$. Samples: compound in the doses of 125, 100, 50, and $25 \mathrm{mg}$, previously incubated with the venom and then added to plasma for time measuring. (B) Effect of the compound 4-(2-chloro-phenyl)-2,7,7-trimethyl-5-oxo1,4,5,6,7,8-hexahydroquinoline-3-carboxylic acid ethyl ester on the coagulation (induced by the venom of Bothrops moojeni) of citrated human plasma. Positive control $(\mathrm{C}+)$ : Bothrops moojeni venom $(40 \mu \mathrm{g})$. Samples: compound in the doses of 125, 100, 50, and $25 \mathrm{mg}$, previously incubated with the plasma and subsequent addition of the venom for time measuring. Data represent the means and standard deviation of triplicates. *Statistically significant - differs from the positive control $(\mathrm{p}<0.05)$.

Thrombi are formed by abnormal control of coagulation factors and clot lysis. Blood coagulation occurs by the activation of intrinsic and extrinsic pathways (19). It is 
important to note that the compound did not present coagulant activity but rather has anticoagulant activity.

When previously incubated with the venom, the compound exerted partial inhibition on proteases. It also interacted with coagulation cascade components when previously incubated with the plasma, interfering with the activation of the factors induced by snake venom proteases. Some enzymes present in the Bothrops spp. venoms can activate coagulation and have a similar action to human thrombin - breakdown of fibrinogen molecules and generating fibrin networks $(20,21)$.

Anticoagulants are used for prophylaxis (preventive healthcare) and treatment of thromboembolic disorders, such as deep vein thrombosis (DVT), pulmonary and systemic embolism, and cerebral ischemia. These disorders are characterized by the formation of blood clots in the vasculature. Anticoagulants that target fibrin formation (e.g., heparin, warfarin, and more recently direct thrombin inhibitors) are mainly used for conditions involving venous blood clots in deep vein thrombosis and pulmonary embolism. They can also be used for people with atrial fibrillation or transient ischemic attack to prevent ischemic stroke (22).

Thrombotic events due to blood clotting are a severe problem in cardiovascular diseases. Although heparin (protease inhibitor) has been used as an anticoagulant to prevent these diseases, its prolonged use may result in the development of thrombocytopenia and an exaggerated response of the immune system. Other drugs such as aspirin and clopidogrel can cause serious adverse effects. Thus, the discovery of new anticoagulant agents would result in alternatives for preventing and treating thrombosis. Cotabarren et al. (23) synthesized, based on natural alkaloids, a cystatin isolated from the plant Moringa oleifera. The authors tested its action as an alternative anticoagulant agent.

\section{Effects on human blood thrombi and venom-induced thrombolytic activity}

The synthesized molecule induced lysis of blood thrombi at all doses tested, which

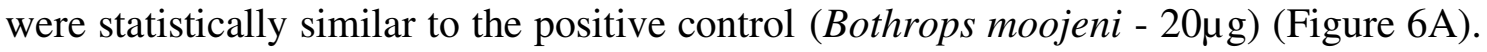
While this result is consistent with the activity observed in the previous experiment, it should be noted that the activities shown here were biologically lower than the control (approximately 20\% difference between $\mathrm{C}+$ and treatments).

When incubating the synthesized compound with $B$. moojeni venom, a significant reduction $(\mathrm{p}<0.05)$ in the venom-induced thrombolytic activity was observed for the 125 
and $100 \mathrm{mg}$ doses (73\% and 79\%, respectively). The other doses were not statistically different from the positive control (Figure 6B).

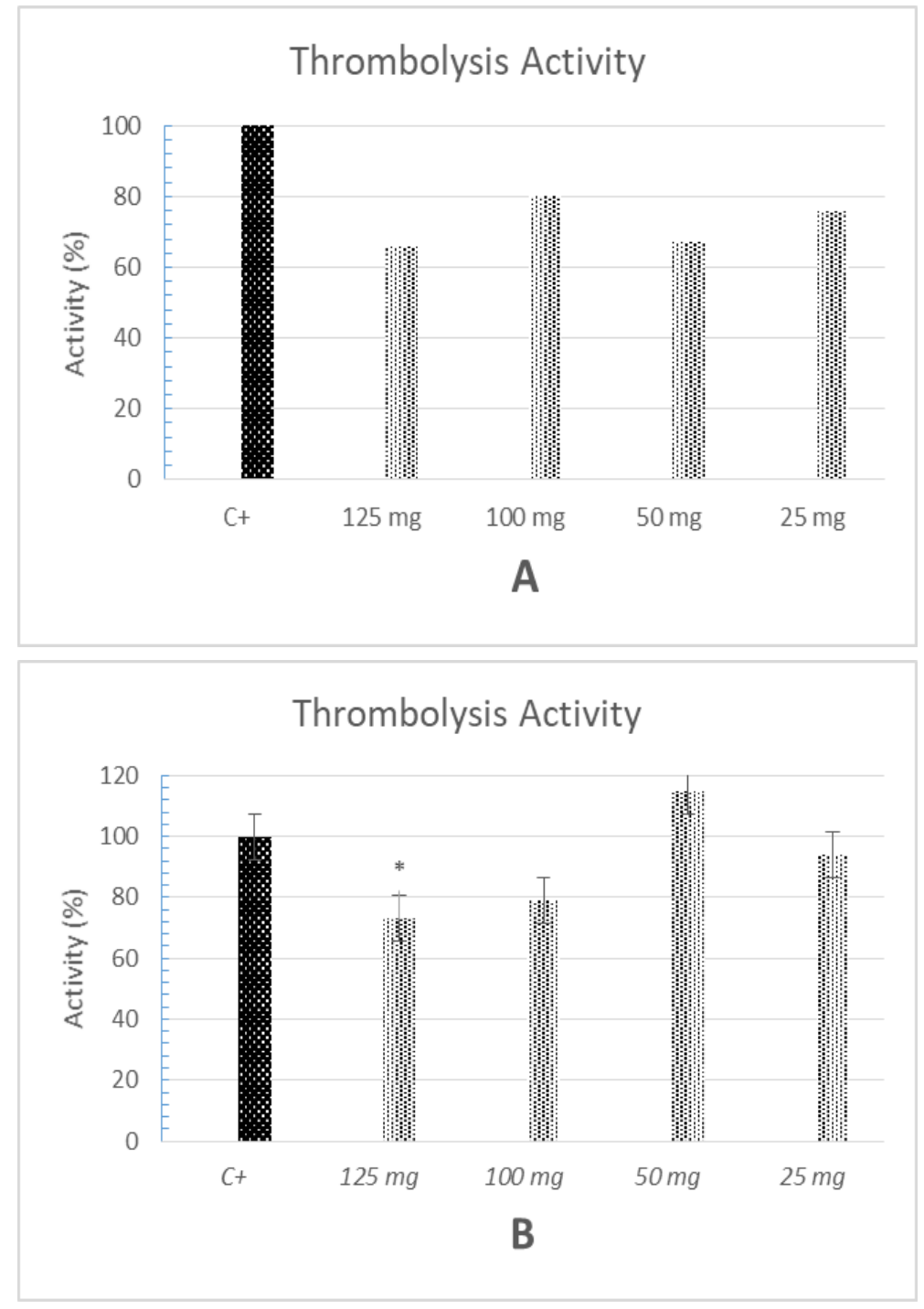

Figure 6. (A) Effect of the compound 4-(2-chloro-phenyl)-2,7,7-trimethyl-5-oxo1,4,5,6,7,8-hexahydroquinoline-3-carboxylic acid ethyl ester on human blood thrombi. Positive control $(\mathrm{C}+)$ : Bothrops moojeni $(20 \mu \mathrm{g})=100 \%$ thrombus dissolution. Samples: compound at 125, 100, 50, and $25 \mathrm{mg}$. (B) Effect of the compound 4-(2-chloro-phenyl)2,7,7-trimethyl-5-oxo-1,4,5,6,7,8-hexahydroquinoline-3-carboxylic acid ethyl ester on the thrombolytic activity induced by proteases from Bothrops moojeni venom. Positive control (C+): Bothrops moojeni venom $(20 \mu \mathrm{g})$. Samples: compound at the doses of 125, 100,50 , and $25 \mathrm{mg}$ previously incubated with the venom and then added to the thrombi. Data represent the means and standard deviation of triplicates. *Statistically significant differs from the positive control $(\mathrm{p}<0.05)$. 
Thus, it is possible to observe that the compound has a thrombolytic activity, which corroborates with the data obtained in the coagulation test using citrated human plasma.

Cardiovascular diseases are caused by disorders of the heart and blood vessels. Nowadays, endogenous thrombi formation in these vessels is one of the leading causes of death. Therefore, many types of research have been performed in the field of prevention and treatment for these diseases (24).

Silva et al. (25) tested the thrombolytic activity of the fibrinolytic enzyme purified from Chlorella vulgaris. They found an estimated lysis rate of $25.6 \%$ after 90 minutes of reaction, while the negative and positive controls showed $3.5 \%$ and $30 \%$, respectively. Drozdov et al. (26) investigated the thrombolytic activity of magnetic polyelectrolytebased composites and showed results of enoxaparin and poly (acrylic acid) -based composites close to pure urokinase (positive control).

The antithrombotic effects of chlorogenic acid were evaluated using the blood clot assay, which is similar to the test performed in the present work. The acid showed a high level of clot degradation compared to the control (19).

It can be inferred from the results obtained in the present work that the synthesized compound has promising thrombolytic characteristics and a protective effect on human plasma components. However, further testing is needed since there are no complementary data in the literature for polyhydroquinoline derivatives.

\section{Effects on the activity of phospholipases $A_{2}$}

The synthesized molecule 4-(2-chloro-phenyl)-2,7,7-trimethyl-5-oxo-1,4,5,6,7,8hexahydroquinoline-3-carboxylic acid ethyl ester, when incubated with Bothrops moojeni venom $(20 \mu \mathrm{g})$, showed inhibitory activity on phospholipases $A_{2}$ at the doses of 125,100 , and $50 \mathrm{mg}(\mathrm{p}<0.05)$. The positive control (crude venom) induced a halo of $16 \mathrm{~mm}$, while the halos formed after incubations with the molecule were $11 \mathrm{~mm}$ for the 125 and $100 \mathrm{mg}$ doses and $13.7 \mathrm{~mm}$ for $50 \mathrm{mg}$ (Figure 7). 


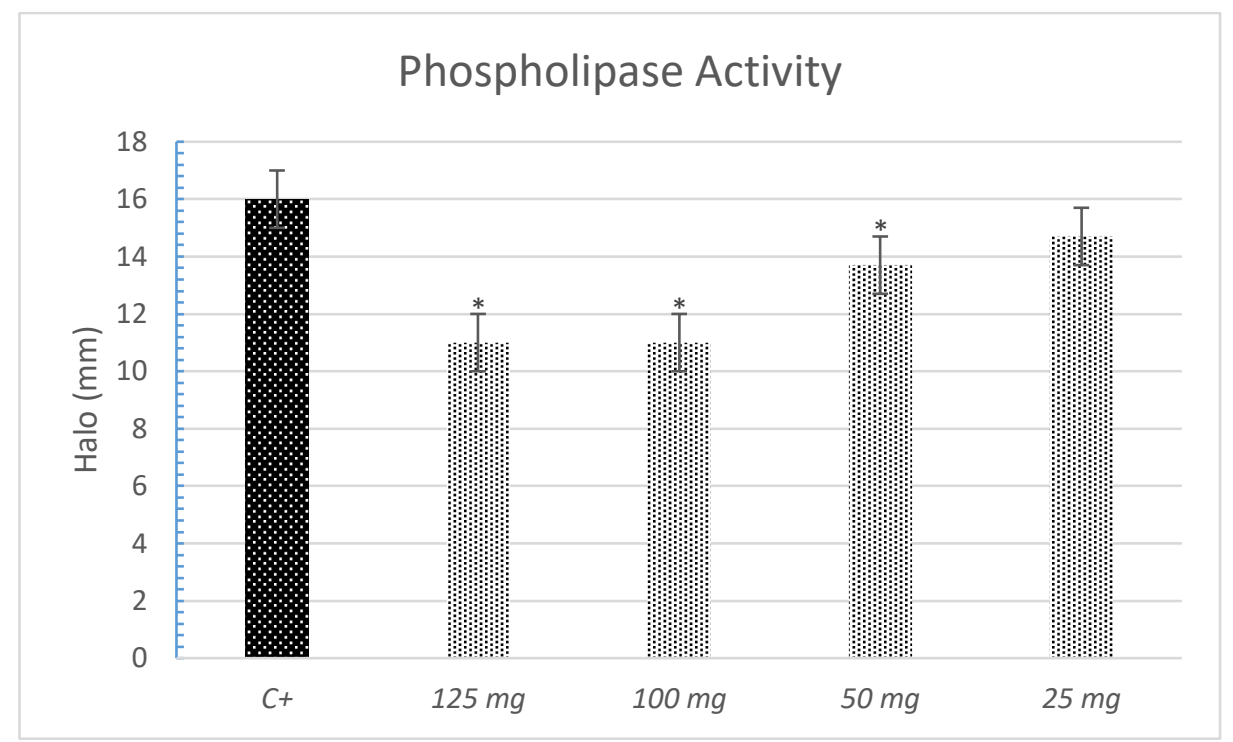

Figure 7. Effect of the compound 4-(2-chloro-phenyl)-2,7,7-trimethyl-5-oxo-1,4,5,6,7,8hexahydroquinoline-3-carboxylic acid ethyl ester on the phospholipase activity induced by Bothrops moojeni venom. Positive control (C+): Bothrops moojeni venom (20 $\mu \mathrm{g})$. Samples: compound at the doses of $125,100,50$, and $25 \mathrm{mg}$, previously incubated with venom. Data represent the means and standard deviation of triplicates. *Statistically significant - differs from the positive control $(\mathrm{p}<0.05)$.

Phospholipases are lipolytic enzymes fundamental to living organisms. The phospholipase $\mathrm{A}_{2}$ family is involved in the cleavage of an sn-2 ester bond of the glycerophospholipid. This breakdown generates 1-acyl-lysophospholipids and free fatty acids, mainly polyunsaturated fatty acids, which are metabolized into bioactive lipid mediators such as prostaglandins and leukotrienes. $\mathrm{PLA}_{2} \mathrm{~S}$ have a fundamental role in inflammation processes and arachidonic acid metabolism in mammals, making the study of these enzymes attractive (27).

Zhao et al. (28) tested the anti-inflammatory potential of three alkaloids and observed activities similar to the commercial drug used as a positive control. Preciado $e t$ al. (29) tested the ethanolic extract of Swietenia macrophylla leaves on the phospholipase $\mathrm{A}_{2}$ from Bothrops asper venom, using egg yolk as the source of phospholipids. The extract exhibited an effect of $87.2 \pm 11 \%$ on neutralizing the enzyme activity. These data found in the literature corroborates the results obtained in the present study with the polyhydroquinoline derivative since they highlight alkaloids with inhibitory action on 
phospholipases $\mathrm{A}_{2}$. Therefore, they are potential candidates for the development of new anti-inflammatory drugs.

Kashid et al. (30) tested some compounds derived from 2,5-disubstituted-1,3,4oxadiazole, which contains a heterocyclic ring structure. The anti-inflammatory activity screening showed promising results compared to diclofenac sodium, used as a positive control.

The anti-inflammatory activity of the molecule N-(3-methoxyphenyl)-3-(pyridin4-yl)-1H-pyrazole-5-carboxamide was also tested. The results were compared to diclofenac sodium (positive control), and the concentrations evaluated (10, 50, 100, 250, and $500 \mu \mathrm{M})$ showed higher anti-inflammatory activity than the control (31).

\section{Cytotoxic activity on human erythrocytes}

4-(2-chloro-phenyl)-2,7,7-trimethyl-5-oxo-1,4,5,6,7,8-hexahydroquinoline-3carboxylic acid ethyl ester did not exhibit cytotoxic activity on human erythrocytes under the conditions and doses tested (data not shown).

However, the synthesized molecule was able to inhibit the activity of cytotoxic proteases present in Bothrops moojeni venom $(20 \mu \mathrm{g})$ in all doses evaluated $(\mathrm{p}<0.05)$. The positive control had an activity halo of $15.5 \mathrm{~mm}$, and the pre-incubation of the venom with doses $125,100,50$, and $25 \mathrm{mg}$ resulted in halos of 8.8, 9.2, 9.6, and $12.1 \mathrm{~mm}$, respectively (Figure 8).

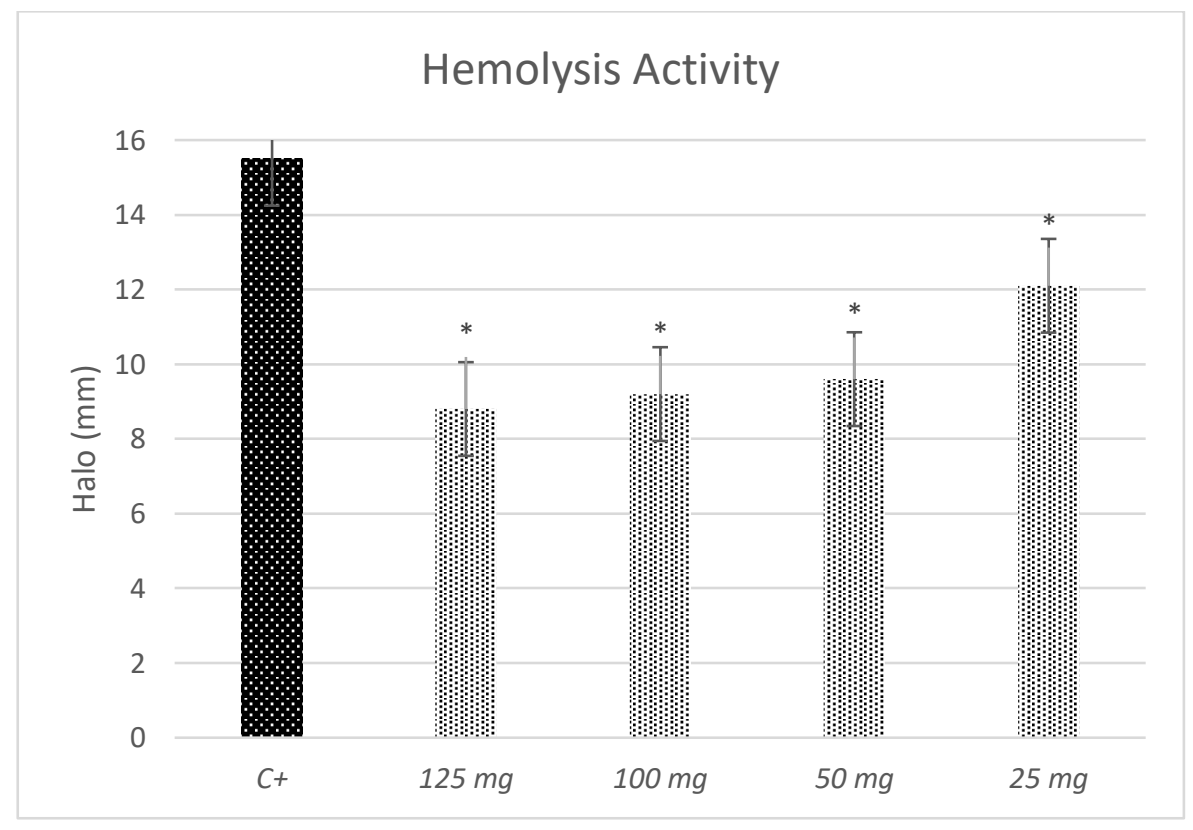


Figure 8. Effect of the compound 4-(2-chloro-phenyl)-2,7,7-trimethyl-5-oxo-1,4,5,6,7,8hexahydroquinoline-3-carboxylic acid ethyl ester on the cytotoxic activity induced by Bothrops moojeni venom in human erythrocytes. Positive control $(\mathrm{C}+)$ : Bothrops moojeni venom $(20 \mu \mathrm{g})$. Samples: compound in the doses of 125, 100, 50, and $25 \mathrm{mg}$, previously incubated with the venom. Data represent the means and standard deviation of triplicates. *Statistically significant - differs from the positive control $(\mathrm{p}<0.05)$.

The erythrocyte membrane is an appropriate and representative model to study the interaction of natural or synthetic compounds with cell membranes. Colina et al. (32) performed tests with different concentrations of epigallocatechin gallate to determine its protective activity on human erythrocyte lysis induced by hypochlorous acid ( $\mathrm{HClO})$. The authors observed progressive inhibition of hemolysis after adding the oxidizing agent, reaching a minimum of $4.5 \%$ hemolysis with $100 \mu \mathrm{M}$ of gallate. The polyhydroquinoline derivative in our study performed the same protection on the red cells membrane.

Diosmin is a flavone glycoside that is naturally found in many citrus fruits. This substance is known to act on the circulatory system's veins, improving blood circulation and venous tonus. In microcirculation, diosmin normalizes permeability and reinforces the resistance of the capillary vessels, which reduces edema. However, despite the broad therapeutic potential of this substance, it is characterized by low solubility in water and most organic solvents. Since diosmin has low bioavailability, high dosages (500 mg) are required for the oral regimen (33).

Therefore, finding new molecules with protective and anti-inflammatory activities is extremely necessary. As can be seen, the molecule 4-(2-chloro-phenyl)-2,7,7trimethyl-5-oxo-1,4,5,6,7,8-hexahydroquinoline-3-carboxylic acid ethyl ester has effects that deserve attention and expanded studies since it is pharmacologically active at a dosage that is considered low when compared to marketed drugs.

\section{CONCLUSION}

In the present work, it was possible to synthesize the polyhydroquinoline derivative 4-(2-chloro-phenyl)-2,7,7-trimethyl-5-oxo-1,4,5,6,7,8-hexahydroquinoline-3carboxylic acid ethyl ester without the use of solvents and catalysts, which is in accordance with green chemistry. The molecule showed anticoagulant, thrombolytic, anti-cytotoxic, and antimicrobial (Gram-positive and Gram-negative bacteria) activities and inhibited the action of phospholipase $\mathrm{A}_{2}$ and thrombolytic proteases in vitro. The 
results obtained are a starting point to further investigations with this molecule and other polyhydroquinoline derivatives. These molecules are promising candidates as active ingredients in the development of new drugs for the treatment of cardiovascular and inflammatory diseases.

\section{Declaration of Competing Interest}

The autors declare that they have no Known competing financial interests or personal relationships that could have appeared to influence the work reported in this paper.

\section{Acknowledgments}

This work was supported by the Fundação de Amparo à Pesquisa de Minas Gerais (FAPEMIG), Coordenação de Aperfeiçoamento de Pessoal de Nível Superior (CAPES) and Conselho Nacional de Desenvolvimento Científico e Tecnológico (CNPq).

\section{REFERENCES}

1. Outili N, Kerras H, Nekkab C, Merouani R, Meniai AH. Biodiesel production optimization from waste cooking oil using green chemistry metrics. Renew Energy [Internet]. 2020;145:2575-86. Available from: https://doi.org/10.1016/j.renene.2019.07.152

2. Naveenkumar R, Baskar G. Process optimization, green chemistry balance and technoeconomic analysis of biodiesel production from castor oil using heterogeneous nanocatalyst. Bioresour Technol [Internet]. 2021;320(PA):1-10. Available from: https://doi.org/10.1016/j.biortech.2020.124347

3. Pinto LMA, Adeoye O, Thomasi SS, Francisco AP, Cabral-Marques H. A singlestep multicomponent synthesis of a quinoline derivative and the characterization of its cyclodextrin inclusion complex. J Mol Struct [Internet]. 2021;1237:1-10. Available from: https://doi.org/10.1016/j.molstruc.2021.130391

4. Dekamin MG, Karimi Z, Latifidoost Z, Ilkhanizadeh S, Daemi H, Naimi-Jamal MR, et al. Alginic acid: A mild and renewable bifunctional heterogeneous biopolymeric organocatalyst for efficient and facile synthesis of polyhydroquinolines. Int J Biol Macromol [Internet]. 2018;108:1273-80. Available from: http://dx.doi.org/10.1016/j.ijbiomac.2017.11.050

5. Nguyen VT, Nguyen HT, Tran PH. One-pot three-component synthesis of 1- 
amidoalkyl naphthols and polyhydroquinolines using a deep eutectic solvent: a green method and mechanistic insight. New J Chem. 2021;45(4):2053-9.

6. Sharma S, Singh UP, Singh AP. Synthesis of MCM-41 supported cobalt (II) complex for the formation of polyhydroquinoline derivatives. Polyhedron [Internet]. 2021;199:1-11. Available from: https://doi.org/10.1016/j.poly.2021.115102

7. Clinical and Laboratory Standards Institute (CLSI). Clinical and Laboratory Standards Institute Methods for Dilution Antimicrobial Susceptibility Tests for Bacteria That Grow Aerobically Standard,, Approval CDM-A.; M07 Methods for dilution Antimicrobial Susceptibility Tests for Bacteria That Grow Aerobically. 2018.

8. CLSI. Performance Standards for Antimicrobial Disk Suspectibility Tests, Approved Standard-Eleventh Edition. Clinical and LAboratory Standards Institue. Vol. 38. 2018.

9. Mourao PAS, Pereira MS, Pavo MSG, Mulloy B, Tollefsen DM, Mowinckel $\mathrm{MC}$, et al. Structure and anticoagulant activity of a fucosylated chondroitin sulfate from echinoderm. Sulfated fucose branches on the polysaccharide account for its high anticoagulant action. J Biol Chem. 1996;271(39):23973-84.

10. Cintra ACO, De Toni LGB, Sartim MA, Franco JJ, Caetano RC, Murakami MT, et al. Batroxase, a new metalloproteinase from B. atrox snake venom with strong fibrinolytic activity. Toxicon [Internet]. 2012;60(1):70-82. Available from: http://dx.doi.org/10.1016/j.toxicon.2012.03.018

11. Gutiérrez J, Avila C, Rojas E, Cerdas L. An alternative in vitro method for testing the potency of the polyvalent antivenom produced in Costa Rica. Toxicon. 1988 Jan;26(4):411-3.

12. Kumar S, Sharma P, Kapoor KK, Hundal MS. An efficient, catalyst- and solventfree, four-component, and one-pot synthesis of polyhydroquinolines on grinding. Tetrahedron. 2008;64(3):536-42.

13. Matada BS, Yernale NG. The contemporary synthetic recipes to access versatile quinoline heterocycles. Synth Commun [Internet]. 2021;51(8):1133-59. Available from: https://doi.org/10.1080/00397911.2021.1876240

14. Perrin CL, Chang KL. The Complete Mechanism of an Aldol Condensation. J Org Chem. 2016;81(13):5631-5.

15. WARREN, Stuart ; WYATT P. Designing Organic Syntheses: a programmed 
introduction to the synthon approach. 2nd ed. New York; 2009. 136 p.

16. Jubeh B, Breijyeh Z, Karaman R. Resistance of gram-positive bacteria to current antibacterial agents and overcoming approaches. Molecules. 2020;25(12):1-23.

17. Jamale DK, Undare SS, Valekar NJ, Sarkate AP, Kolekar GB, Anbhule P V. Glycerol Mediated Synthesis, Biological Evaluation, and Molecular Docking Study of 4-(1H-pyrazol-4-yl)-polyhydroquinolines as Potent Antitubercular Agents. J Heterocycl Chem. 2019;56(2):608-18.

18. Mane P, Shinde B, Mundada P, Karale B, Burungale A. Biogenic synthesis of $\mathrm{ZnO}$ nanoparticles from Parthenium histerophorus extract and its catalytic activity for building bioactive polyhydroquinolines. Res Chem Intermed [Internet]. 2021;47(5):1743-58. Available from: https://doi.org/10.1007/s11164020-04365-8

19. Choi J. Investigation of the anticoagulant and antithrombotic effects of chlorogenic acid. J Biochem Mol Toxicol. 2016;31(September):1-6.

20. Sitprija V, Sitprija S. Renal effects and injury induced by animal toxins. Toxicon [Internet]. 2012;60(5):943-53. Available from: http://dx.doi.org/10.1016/j.toxicon.2012.06.012

21. Albuquerque PLMM, Paiva JHHGL, Martins AMC, Meneses GC, Da Silva GB, Buckley N, et al. Clinical assessment and pathophysiology of Bothrops venomrelated acute kidney injury: A scoping review. J Venom Anim Toxins Incl Trop Dis. 2020;26:1-17.

22. Fischer PM. Design of Small-Molecule Active-Site Inhibitors of the S1A Family Proteases as Procoagulant and Anticoagulant Drugs. J Med Chem. 2018;61(9):3799-822.

23. Cotabarren J, Claver S, Payrol JA, Garcia-Pardo J, Obregón WD. Purification and characterization of a novel thermostable papain inhibitor from moringa oleifera with antimicrobial and anticoagulant properties. Pharmaceutics. 2021;13(4):1-11.

24. Brodmann M, Cahill TJ, Hil DP, Carapetis J, Catapano AL, Chugh SS, et al. Global Burden of Cardiovascular Diseases. J Am Coll Cardiol. 2020;76(25):2982-3021.

25. Silva PE da C e., Barros RC de, Albuquerque WWC, Brandão RMP, Bezerra RP, Porto ALF. In vitro thrombolytic activity of a purified fibrinolytic enzyme from Chlorella vulgaris. J Chromatogr B Anal Technol Biomed Life Sci [Internet]. 
2018;1092(January):524-9. Available from:

https://doi.org/10.1016/j.jchromb.2018.04.040

26. Drozdov AS, Prilepskii AY, Koltsova EM, Anastasova EI, Vinogradov V V. Magnetic polyelectrolyte-based composites with dual anticoagulant and thrombolytic properties: towards optimal composition. J Sol-Gel Sci Technol [Internet]. 2020;95(3):771-82. Available from: http://dx.doi.org/10.1007/s10971020-05313-Z

27. Peggion C, Tonello F. Short linear motifs characterizing snake venom and mammalian phospholipases a2. Toxins (Basel). 2021;13(4):1-15.

28. Zhao L, Li W, Dai SJ, Liu RX, Xie ZP, Zhang SM, et al. Alkaloids bearing rare skeletons from Forsythia suspensa with anti-inflammatory and anti-viral activities in vitro. Phytochemistry [Internet]. 2021;186(November 2020):1-7. Available from: https://doi.org/10.1016/j.phytochem.2021.112739

29. Preciado L, Pereañez JA, Nuñez V, Lobo-Echeverri T. Characterization of the most promising fraction of Swietenia macrophylla active against myotoxic phospholipases A2: Identification of catechin as one of the active compounds. Vitae. 2016;23(2):124-33.

30. Kashid BB, Salunkhe PH, Dongare BB, More KR. Bioorganic \& Medicinal Chemistry Letters their in vitro anti-inflammatory, anti-oxidant evaluation , and molecular docking study. Bioorg Med Chem Lett [Internet]. 2020;30(12):127136. Available from: https://doi.org/10.1016/j.bmcl.2020.127136

31. Nithyabalaji R, Krishnan H, Sribalan R. Synthesis , molecular structure and multiple biological activities of N-. J Mol Struct [Internet]. 2019;1186:1-10. Available from: https://doi.org/10.1016/j.molstruc.2019.02.095

32. Colina JR, Suwalsky M, Manrique-Moreno M, Petit K, Aguilar LF, JemiolaRzeminska M, et al. Protective effect of epigallocatechin gallate on human erythrocytes. Colloids Surfaces B Biointerfaces [Internet]. 2019;173:742-50. Available from: https://doi.org/10.1016/j.colsurfb.2018.10.038

33. Szeleszczuk Ł, Pisklak DM, Zielińska-Pisklak M, Wawer I. Spectroscopic and structural studies of the diosmin monohydrate and anhydrous diosmin. Int $\mathbf{J}$ Pharm. 2017;529(1-2):193-9. 